\title{
CICLO DE VIDA DE Dasineura gigantea ANGELO \& MAIA, 1.999 (DIPTERA, CECIDOMYIIDAE)
}

\author{
Alessandro Camargo Angelo* \\ *Eng. Florestal, Dr., Depto. de Ciências Florestais, UFPR - alessandro.angelo@ufpr.br \\ Recebido para publicação: 23/02/2006 - Aceito para publicação: 17/05/2007
}

\begin{abstract}
Resumo
Dasineura gigantea induz a galha dos botões em araçazeiro (Psidium cattleianum, Myrtaceae), planta nativa do Brasil que foi introduzida em algumas áreas tropicais do mundo. As galhas são histióides, prosoplasmáticas e multiloculares $(3,45 \pm 2,33$ câmaras por galha, $\mathrm{n}=260)$, com crescimento iniciado em duas épocas: fevereiro/abril e agosto/outubro. As fêmeas depositam ovos sobre os tecidos da planta e a alimentação das larvas induz a formação de galhas (período larval: 152,27 $\pm 9,35$ dias, $\mathrm{n}$ = 86). As larvas possuem uma espátula protorácica que é usada para abrir o orifício de saída do adulto. Após a construção desse orifício, a larva abriga-se no interior da galha e empupa (período pupal: $35,67 \pm 1,62 \mathrm{dia}, \mathrm{n}=27$ ). Com o desenvolvimento do adulto, o inseto contorce-se e força sua saída através do orifício de emergência, deixando o tegumento pupal preso a esse orifício. Os adultos não se alimentam e tem um período de vida curto (um dia, $\mathrm{n}=45$ ), apenas o tempo necessário para o acasalamento e oviposição.

Palavras-chave: Galhas; Cecidomyiidae; Dasineura; Myrtaceae.
\end{abstract}

\begin{abstract}
Life history of Dasineura gigantea Angelo \& Maia, 1.999 (Diptera, Cecidomyiidae). Dasineura gigantea induces bud-galls on the strawberry-guava (Psidium cattleianum, Myrtaceae), native plant of Brazil that was introduced in some tropical areas of the world. The galls are histioid, prosoplasmic and multiloculate $(3,45 \pm 2,33$ chambers for gall, $\mathrm{n}=260)$, and have growth initiated at two times: February / April and August / October. The females deposit eggs on the plant tissue and the feeding of the larvae induces the formation of galls (larval period: $152,27 \pm 9,35$ days, $n=86$ ). These larvae show prothoracic spatula, used to open an orifice of exit for the adult. After the construction of this orifice, the larva searchs shelter in the interior of the gall and changed itself into pupae (pupal period: $35,67 \pm 1,62$ days, $\mathrm{n}=27$ ). With the development of the completed adult, the insect wriggles and forces its exit through the exit orifice, leaving the pupal skin imprisoned to this orifice. The adults do not feed themselves and have a short period of life (one day, $n=45$ ), only the necessary time for mating and oviposition.

Keywords: Gall; Cecidomyiidae; Dasineura; Myrtaceae.
\end{abstract}

\section{INTRODUÇÃO}

Para Mani (1992), as galhas representam uma série complexa de interações entre os tecidos de uma planta e outro organismo vivo. A compreensão dessas interações envolve estudos sobre a estrutura das galhas, sua citologia, bioquímica e fisiologia. Entre os principais grupos de indutores de galhas, Dreger-Jauffret e Shorthouse (1992) relacionam a família Cecidomyiidae (Diptera).

O gênero Dasineura Rondani 1840, de acordo com Gagné (1989), é o maior em Cecidomyiidae, com 110 espécies conhecidas na região Neártica. Gagné (1968) citava apenas sete espécies desse gênero para a região Neotropical, uma delas para o Brasil, D. brasiliensis, que havia sido descrita por Tavares (1922).

Posteriormente, outras espécies foram descritas no Brasil, como, por exemplo, $D$. copacabanensis associada a Eugenia spp (Myrtaceae), em Maia (1993). Como exemplo de trabalhos descrevendo novas espécies no Brasil pode-se citar ainda Maia (2001), Maia et al. (2002) e Madeira et al.

FLORESTA, Curitiba, PR, v. 38, n. 1, jan./mar. 2008. 
(2003). Ângelo; Maia (1999) descreveram uma nova espécie pertencente ao gênero, D. gigantea, indutora de galhas em Psidium cattleianum Sabine (Myrtaceae), espécie conhecida popularmente como araçazeiro, de acordo com Legrand; Klein (1977).

Apesar desses trabalhos citarem esse gênero na região neotropical, poucos trabalhos sobre o ciclo de vida e sua ecologia foram realizados (Andrade et al., 1995). Um exemplo de interação entre $P$. cattleianum e um indutor de galhas, Neotrioza tavaresi Crawford, 1925 (Hemiptera, Psyllidae), foi registrado por Butignol; Pedrosa-Macedo (2003).

Neste trabalho, foram realizadas observações visando o conhecimento do ciclo de vida de $D$. gigantea no que se refere à duração de suas fases larval, pupal e adulta, além do número de ovos por postura.

P. cattleianum é considerada uma planta indesejável em várias partes do mundo (Yahaya, 2003; Meyer, 2004 e Wirth et al., 2004), entre elas o arquipélago do Havaí, conforme enfatizado por Hodges (1988), Hueneck e Vitousek (1990) e Markin et al. (1991).

Por outro lado, $D$. gigantea é um de seus inimigos naturais, devido às galhas causadas, o que torna a espécie interessante para estudos que buscam agentes de controle biológico para $P$. cattleianum. Quanto a esse aspecto, Julien (1993) relaciona algumas espécies pertencentes ao gênero Dasineura que foram empregadas em programas de controle biológico de plantas indesejáveis. Existem diversos exemplos de cecidomyiidae relacionados a programas de controle biológico de plantas (SKUHRAVA; HINZ, 2000; SOBHIAN et al., 2000; SYRETT et al., 2001; GAGNÉ et al., 2004; e LLOYD et al., 2005).

\section{MATERIAL E MÉTODOS}

Foram definidas duas áreas para acompanhamento e coleta de galhas, escolhidas em função da abundância de exemplares de $P$. cattleianum com galhas induzidas por $D$. gigantea. O período de coleta foi de novembro de 1994 a janeiro de 1997 . A primeira área $\left(25^{\circ} 29^{\prime} 52^{\prime \prime} \mathrm{S}\right.$ e $\left.48^{\circ} 58^{\prime} 52^{\prime \prime} \mathrm{W}\right)$, com altitude de 1021 metros, localiza-se no município de Piraquara (PR), em área de transição de Floresta Ombrófila Mista para Floresta Ombrófila Densa (RODERJAN et al., 1993). A segunda área (2605'17” S e 48³7’36” W) localiza-se no município de Itapoá (SC), em área de restinga (IBGE, 1988; VELOSO et al. 1991). Em ambas as áreas, as plantas ocorriam em clareiras, em função do caráter heliófilo dessa espécie (LEGRAND; KLEIN, 1977).

Os dados do ciclo de vida foram obtidos pelo acompanhamento da evolução do agente indutor da galha através de observações em campo, as quais foram confrontadas com informações de amostras dissecadas e mantidas em laboratório.

No total, 60 galhas foram mantidas em campo(sendo 30 por local), etiquetadas e envoltas com tecido do tipo "voil" (sacos com 18 centímetros de comprimento x 13 centímetros de diâmetro). Outras 10 galhas foram coletadas manualmente, durante 13 meses, em cada um dos locais, totalizando 260 unidades. Estas, assim que foram retiradas das árvores, foram colocadas em recipientes plásticos $(62,83$ $\mathrm{cm}^{3}$ ) com papel de filtro e acondicionadas e transportadas em caixa térmica. Sobre cada recipiente foram feitas anotações de procedência, data, coletor e eventuais observações. O material entomológico foi mantido em câmaras climatizadas com fotofase de 12 horas e temperatura de $25^{\circ} \pm 1^{\circ} \mathrm{C}$, para acompanhamento do ciclo.

As galhas foram parcialmente seccionadas, de maneira a permitir momentaneamente a visualização do interior das câmaras, com a finalidade de se observar o desenvolvimento das formas imaturas. A cada recipiente foi acrescentada uma etiqueta de identificação e papel de filtro umedecido, tal como em Arduin et al. (1994), Kraus et al. (1996) e Maia; Monteiro (1999). Após a constatação da emergência, os adultos foram transferidos para recipientes de vidro com $28 \times 22 \times 25 \mathrm{~cm}$, cobertos com "voil", no interior dos quais foram colocadas mudas de $P$. cattleianum, com $10 \mathrm{~cm}$ na parte epígea, em sacos plásticos de $17 \mathrm{~cm}$ de altura x $8 \mathrm{~cm}$ de diâmetro. No interior desses recipientes, foi acrescentado algodão umedecido em uma mistura de água + glucose, conforme Andrade et al. (1995). 
As dissecações foram realizadas sob lupa, através da abertura das câmaras feita com estiletes e pinças entomológicas. Com essa operação, foram obtidas informações como a extensão do período larval e pupal. Outros parâmetros biológicos quantificados foram a longevidade dos adultos e o número de ovos depositados por indivíduo.

As exsicatas de P. cattleianum foram depositadas no Museu do Departamento de Botânica do Setor de Ciências Biológicas da UFPR, sob número 29.492. Lâminas com exemplares de D. gigantea encontram-se depositadas no Museu de Zoologia da UFPR. A montagem dos cecidomídeos seguiu o método de Gagné (1989).

\section{RESULTADOS E DISCUSSÃO}

Os indivíduos de $D$. gigantea dependem da disponibilidade de gemas foliares em $P$. cattleianum para formarem as galhas, situação observada para outras espécies por Dreger-Jauffret e Shorthouse (1992) e Rohfritsch (1992). Em função disso, existe a necessidade de um sincronismo entre esses insetos e a planta, ou seja, sua sobrevivência depende da formação das galhas (Figura 1), e estas apenas se formam em determinados tecidos da planta, que surgem em períodos restritos. Esse sincronismo envolvendo as galhas e seus indutores foi destacado por Mani (1964), Fernandes et al. (1987), Suárez e Calvo (1989), Ngakan e Yukawa (1997) e Yukawa (2000). Skuhravá e Skuhravý (1992) mencionam situação semelhante envolvendo os galhadores Giraudiella inclusa (Frauenfield), Lasiopetera arundinis Schiner, 1854, L. hungarica Möhn, 1968 e Microlasioptera flexuosa (Winnertz, 1853) (Cecidomyiidae) sobre Phragmites communis Trin. (Graminae).

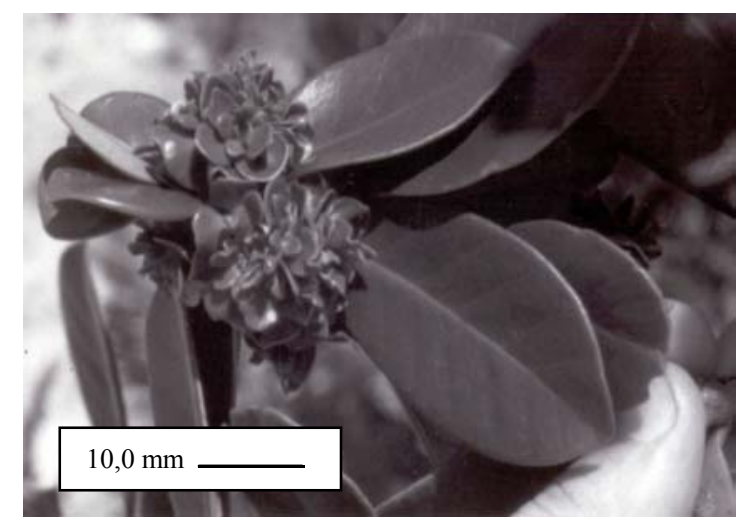

Figura 1. Galha de D. gigantea.

Figure 1. Gall of D. gigantea.

A origem das galhas sobre gemas axilares e botões florais deve-se à capacidade de crescimento desses tecidos, ou seja, pela sua multiplicação celular, razão pela qual respondem melhor ou podem responder à ação do(s) inseto(s), redirecionando essa capacidade de crescimento. A indução de galhas sobre tecidos meristemáticos foi destacada por Fernandes (1987) e por Gagné (1989).

Em função de sua morfologia, as galhas de $D$. gigantea sobre $P$. cattleianum são enquadradas no grupo das galhas histióides, ou seja, aquelas em que há formação de tecidos anormais causada por hipertrofia e/ou hiperplasia celular. Por sua vez, nesse grupo, são consideradas como prosoplasmáticas, por apresentarem forma e tamanho específicos, com diferenciação de tecidos bem definida. Os termos histióide e prosoplasmática foram propostos por Küster (1911), sendo essa nomenclatura considerada por Mani (1964), Arevallo; Romero (1987) e Dreger-Jauffret; Shorthouse (1992). A formação de galhas sobre gemas foliares originando estruturas semelhantes ao deste exemplo foram relatadas por DregerJauffret; Shorthouse (1992), considerando que para a formação dessas galhas os insetos utilizam o potencial de crescimento vegetativo, interrompendo o prolongamento dos internós e a multiplicação de folhas, resultando nas chamadas "rosette galls". 
Em relação ao ciclo de vida, constatou-se que $D$. gigantea é bivoltina, ou seja, apresenta normalmente duas gerações por ano, iniciando-se predominantemente nos meses de agosto/outubro e fevereiro/abril. Deve-se ressaltar que há exemplos no gênero, como D. affinis Kieffer, 1886, que tem seu tempo de ciclo de vida em dependência das estações, registrando desde uma até cinco gerações anuais (BIRCH et al., 1992). Gagné (1994), por sua vez, considera que algumas espécies fitófagas de Cecidomyiidae podem ter um número limitado de gerações por ano, para coincidir com o ciclo de seus hospedeiros.

No caso de $D$. gigantea, logo no primeiro dia de vida dos adultos ocorre o acasalamento, após o qual os machos morrem, enquanto que as fêmeas passam a procurar um local adequado para postura e, assim que o fazem, também morrem. Essas observações foram registradas em condições de laboratório.

Situações semelhantes foram observadas por Ehler; Kinsey (1991) em relação a Rhopalomyia californica Felt, 1908 (Dip., Cecidomyiidae), que induzem galhas sobre Baccharis pilularis D.C. (Asteraceae), e por Stiling et al. (1992), em relação a Asphondylia borrichiae Rossi e Strong 1990 (Dip., Cecidomyiidae) sobre Borrichia frutescens (L.) (Asteraceae).

Foram observadas treze posturas em laboratório (Figura 2), realizadas em gemas axilares na base de pecíolos, constatando-se uma média de $92,24 \pm 25,58$ ovos/postura $(n=17)$. As galhas tornam-se perceptíveis entre a terceira e a quarta semanas após a postura. A princípio, essas galhas surgem como pequenas deformações ou protuberâncias nas gemas axilares e foliares das plantas ou, excepcionalmente, em receptáculos e botões florais. Exemplos semelhantes de estudos compreendendo o período de oviposição, a sobrevivência das larvas e o surgimento das galhas podem ser encontrados em Larsson; Strong (1992) envolvendo Dasineura marginemtorquens Bremi, 1847 (Dip., Cecidomyiidae) sobre Salix viminalis L. (Salicaceae), e em Crook et al. (2001), envolvendo Dasineura tetensi Rübsaamen, 1891 sobre Ribes nigrum L. (Saxifragaceae).

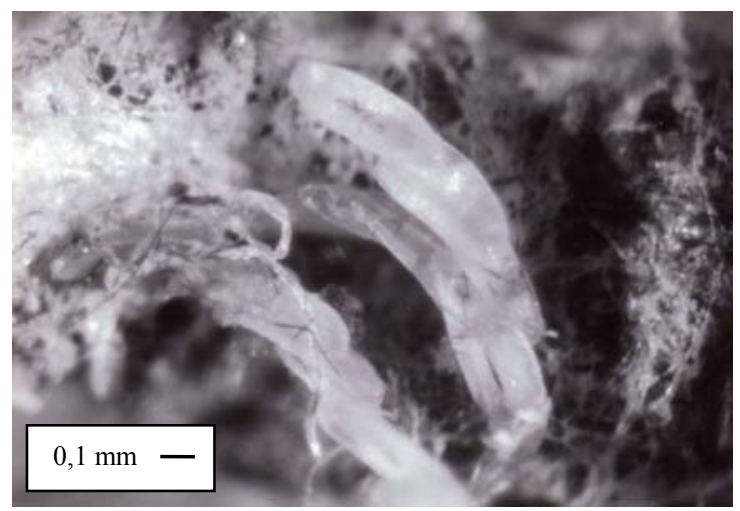

Figura 2. Ovos de D. gigantea.

Figure 2. Eggs of D. gigantea.

Após esse período, as galhas crescem rapidamente em volume, tornando-se mais perceptíveis em campo. As larvas (Figura 3) permanecem no interior das galhas (Figura 4), onde se alimentam até apresentarem a espátula esternal (Figura 5). Com essa estrutura, constroem um canal nos tecidos da galha. Esse canal servirá futuramente para a emergência dos adultos (Figura 6), conforme Gagné (1994).

A existência desse canal possibilitou observar o interior das câmaras para verificar se a camada de tecidos que as envolvem estava ou não rompida e, dessa forma, constatar se o inseto estava presente ou não na câmara. Situação semelhante foi constatada por Stiling et al. (1992), que consideraram a presença de orifícios nas galhas de Asphondylia borrichiae Rossi e Strong (Dip., Cecidomyiidae) sobre Borrichia frutescens (L.) DC (Asteraceae), ressaltando que eles podem diagnosticar a emergência de insetos adultos, tanto do indutor como dos parasitóides. Outros exemplos semelhantes foram constatados por Brewer et al. (1984), estudando Monarthropalpus buxi (Laboulbéne) (Dip., Cecidomyiidae), e por Diatloff; Palmer (1987), em relação a Neolasioptera lathami Gagné, 1971 (Dip., Cecidomyiidae). 


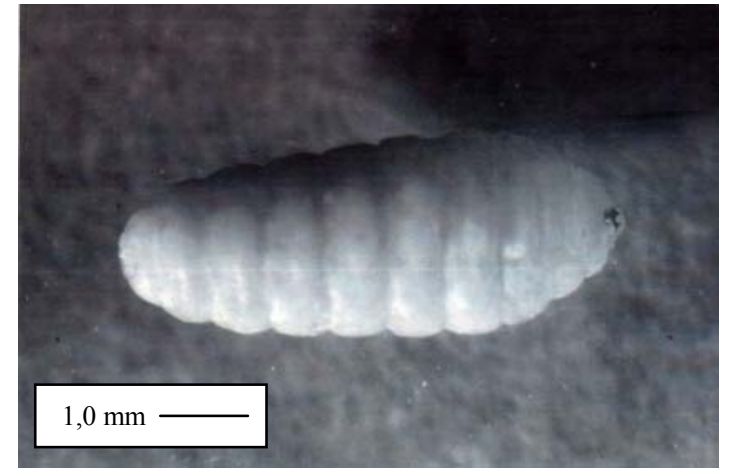

Figura 3. Larva de D.gigantea.

Figure 3. Larvae of D. gigantea.

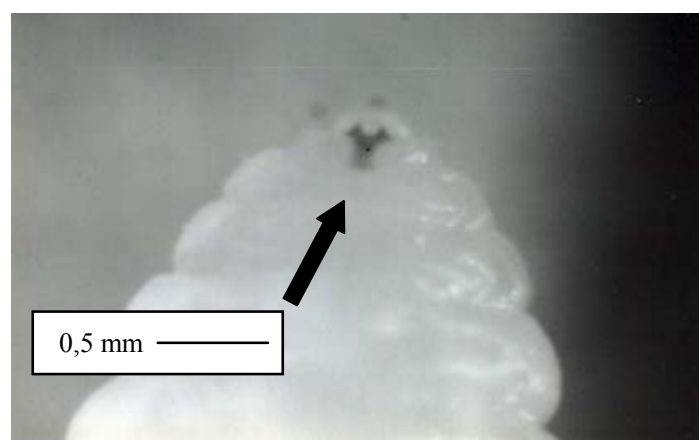

Figura 4. Larvas em câmaras na galha. Figure 4. Larvae in chambers of gall.

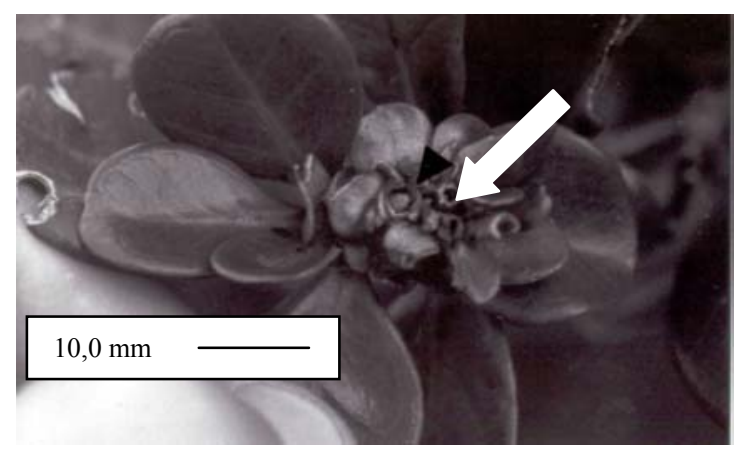

Figura 5. Detalhe da espátula em D. gigantea.Figura 6. Orifício de saída de $D$. gigantea.

Figure 5. Detail of spatula of D. gigantea.

Após a construção do canal, as larvas recolhem-se ao interior de suas câmaras individuais (Figura 7) e empupam. Nas situações observadas, constatou-se que o empupamento ocorreu 152,27 \pm 9,35 dias $(n=43)$ após a realização da postura pelas fêmeas. Em função dessa paralisação da atividade das larvas, as galhas têm suas atividades alteradas, perdendo a sua pigmentação e tornando-se lenhosas, conforme verificado para outras espécies de insetos indutores de galhas por Fernandes et al. (1988), Arduin et al. (1994), Andrade et al. (1995) e Arduin e Kraus (1995). Esse fenômeno foi relatado porque as pupas (Figura 08) só foram encontradas em galhas lenhosas, nas quais haviam canais já construídos. $\mathrm{O}$ período pupal foi de 35,67 $\pm 1,62$ dias $(\mathrm{n}=27)$. A emergência dos imagos fica evidenciada pela presença do tegumento pupal (Figura 9), que permanece preso ao estreito orifício de saída construído pela larva (Figura 10), conforme verificado em Arduin et al. (1991) em estudo envolvendo um tipo de galha sobre Struthantus vulgaris Mart. (Loranthaceae).

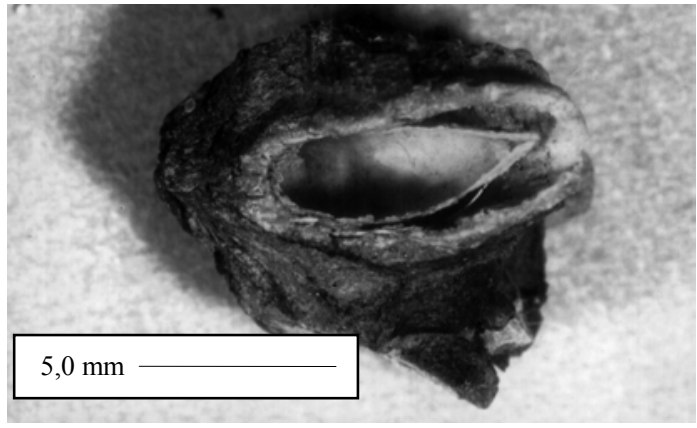

Figura 7. Aspecto da câmara de D. gigantea. Figure 7. Aspect of chamber of D. gigantea.

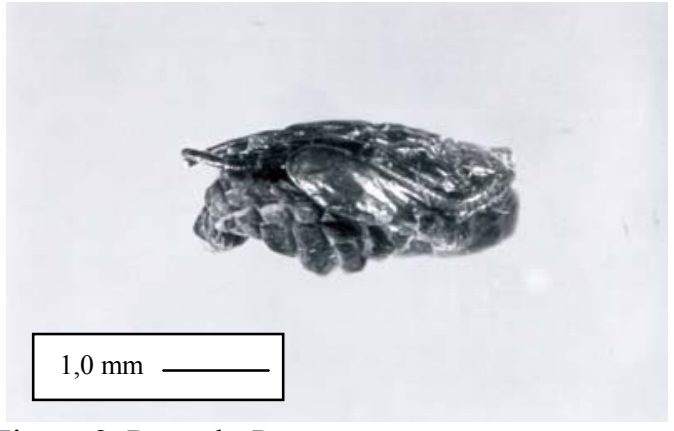

Figura 8. Pupa de D. gigantea. Figure 8. Pupa of D. gigantea. 


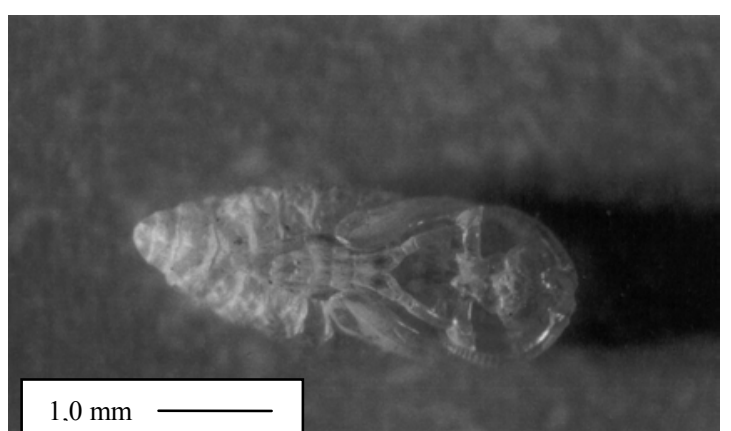

Figura 9. Tegumento pupal de D. gigantea.

Figure 9. Pupal skin of D. gigantea.

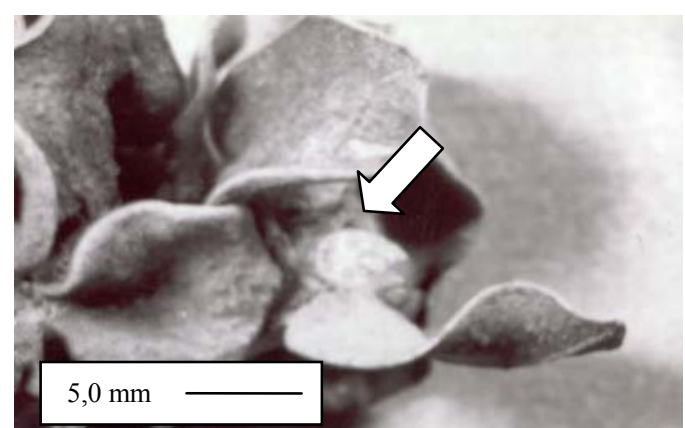

Figura 10. Tegumento preso ao orifício de saída na galha.

Figure 10. Pupal skin restrained in the exit orifice of gall.

A obtenção de adultos (Figura 11) foi conseguida através da emergência deles a partir de galhas coletadas nos meses de fevereiro, março, abril, agosto, setembro e outubro. Nesses meses, uma parte das larvas já havia empupado, ou seja, estava usando a galha como abrigo e não tinha uma relação de alimentação com os tecidos vegetais ao seu redor. Galhas coletadas nos outros meses e armazenadas não propiciaram a obtenção de adultos. Isso ocorreu em função de que, nesses meses, os insetos encontravamse predominantemente na fase larval, na qual apresentam dependência da atividade da planta e, uma vez que as galhas foram retiradas da planta, não puderam concluir seu ciclo.

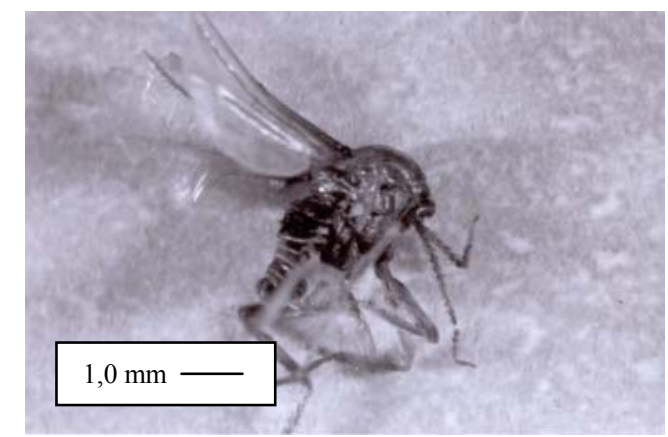

Figura 11. Adulto de D. gigantea.

Figure 11. Adult of D. gigantea.

O período de vida dos adultos é de apenas um dia $(n=45)$, tanto para machos como para fêmeas. Esse período é suficiente para a realização do acasalamento e da postura. De fato, o período de vida dos adultos de muitas espécies da família Cecidomyiidae é efêmero, conforme observação de Gagné (1994), chegando ao ponto desses insetos, em sua fase adulta, não se alimentarem, com exemplos até de espécies com aparelho bucal não-funcional. Esse período curto de vida do adulto também é mencionado por Gagné (1989), que considera que a maior parte dos adultos de hábito fitófago da família Cecidomyiidae sobrevive por apenas um ou dois dias. Na figura 12, estão exemplificados dois períodos com ciclos de vida de $D$. gigantea, ressaltando-se que esses foram os períodos predominantes no período de estudo. O primeiro desses ciclos teve início em meados de março, com o ciclo seguinte tendo início em meados de setembro. 

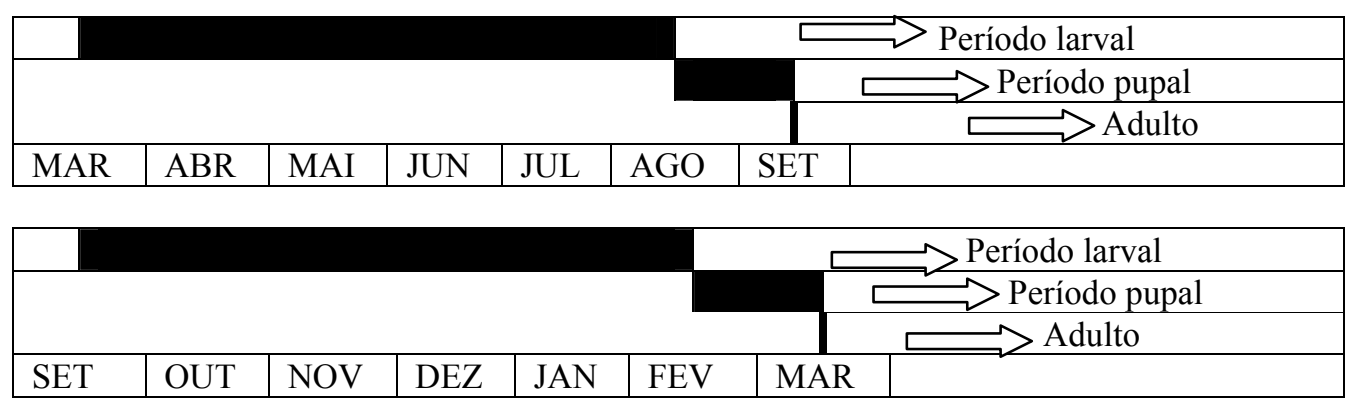

Figura 12. Exemplos de ciclo de vida de $D$ gigantea observados em dois períodos do ano. Figure 12. Examples of cycle of life of $D$. gigantea observed in two periods of the year.

\section{CONCLUSÃO}

O ciclo de $D$. gigantea sobre $P$. cattleianum é bivoltino, com início de ciclo concentrado em duas épocas do ano, a primeira delas de fevereiro a abril, e a segunda, de agosto a outubro. O êxito na formação de galhas depende da sincronização entre a emergência dos insetos e a disponibilidade de tecidos meristemáticos da planta. As galhas ocasionadas são histióides e prosoplasmáticas, sendo induzidas predominantemente sobre gemas foliares de P. cattleianum.

\section{AGRADECIMENTOS}

Ao professor Dr. José Henrique Pedrosa-Macedo (UFPR), ao Dr. Clifford Smith (University of Manoa, Hawaii), ao convênio "Fupef-Universidade do Havaí" e ao CNPq, pelo apoio para a realização do trabalho.

\section{REFERÊNCIAS}

ANDRADE, G. I.; SILVA, I. M.; FERNANDES, G. W.; SCATENA, V. L. Aspectos biológicos das galhas de Tomoplagia rudolphi (Diptera: Tephritidae) em Vernonia polyanthes (Asteraceae). Revista Brasileira de Biologia, Rio de Janeiro, v. 55, n. 4, p. 819-829, 1995.

ANGELO, A. C.; MAIA. V. C. Dasineura gigantea sp. n. (Diptera, Cecidomyiidae) associada a Psidium cattleianum Sabine (Myrtaceae) no Brasil. Revista Brasileira de Zoologia, São Paulo, v. 16, n. 1, p. 191-195, 1999.

ARDUIN, M.; KRAUS, J. E.; VENTURELLI, M. Estudo morfológico de galha achatada em folha de Struthanthus vulgaris Mart. (Loranthaceae). Revista Brasileira de Botânica, São Paulo, v. 14, p. 147156, 1991.

ARDUIN, M.; KRAUS, J. E.; MONTENEGRO, G. Morfologia e fenologia de galhas foliares de Piptadenia gonoacantha (Fabales, Mimosaceae). Revista Brasileira de Entomologia, São Paulo, v. 38, n. 1, p. 79-89, 1994.

ARDUIN, M.; KRAUS, J. E. Anatomia e ontogenia de galhas foliares de Piptadenia gonoacantha (Fabales, Mimosaceae). Boletim de Botânica da Universidade de São Paulo, v. 14, p. 190-130, 1995.

AREVAllo, I. S.; ROMERO, J. H. T. Agallas e insectos associados. In: Plantas de la familia Compositae en flora espontanea del departamento de cundinamarca (Colombia). Bogotá: Universidad Nacional de Colombia, 1987. p. 171-184,

BIRCH, M. L.; BREWER, J. W.; ROHFRITSCH, O. Biology of Dasineura affinis (Cecidomyiidae) and influence of its gall on Viola odorata. In: SHORTHOUSE, J. D.; O. ROHFRITSCH. Biology of insect inducing galls. New York: Oxford University Press, 1992. 285 p. 
BREWER, J. W.; SKUHRAVÝ, V.; SKUHRAVÁ, M. Biology, distribution and control of Monarthropalpus buxi (Laboulbène) (Diptera, Cecidomyiidae). Z. Ang. Ent., Klimakunde, v. 97, p. 167$175,1984$.

BUTIGNOL, C. A.; PEDROSA-MACEDO, J. H. Biology of the leaf gall inducer Neotrioza tavaresi Crawford, 1925 (Hemiptera, Psyllidae) on strawberry guava tree (Psidium cattleianum). Revista Brasileira de Entomologia, São Paulo, v. 47, n. 1, p. 1-7, 2003.

CROOK, D. J.; CROSS, J.; BIRCH, A. N. E.; BRENNAN, R. M.; MORDUE, A. J. Oviposition and larval survival of Dasineura tetensi on four blackcurrant Ribes cultivars. Entomologia Experimentalis et Applicata, Dordrecht, v. 101, n. 2, p. 183-190, 2001.

DIATLOFF, G.; PALMER, W. A. The host specificity of Neolasioptera lathami Gagné (Diptera: Cecidomyiidae) with notes on its biology and phenology. Proceedings of the Entomological Society of Washington, Washington, DC, v. 89, n. 1, p. 122-125, 1987.

DREGER-JAUFFRET, F.; SHORTHOUSE, J. D. Diversity of gall-inducing insects and their galls. p. 833. In: SHORTHOUSE, J. D.; O. ROHFRITSCH. Biology of insect inducing galls. New York: University Press, 1992. 285 p.

EHLER, L. E.; KINSEY, M. G. Ecological recovery of a gall midge and its parasit guild following disturbance. Environmental Entomology, College Park, Md., v. 20, n. 5p. 1295-1300, 1991.

FERNANDES, G. W. Gall forming insets: their economic importance and control. Revista Brasileira de Entomologia, São Paulo, v. 31, n. 3, p. 379-398, 1987.

FERNANDES, G. W.; MARTINS, R. P.; TAMEIRÃO NETO, E. Food web relationships involving Anadiplosis sp. galls (Diptera: Cecidomyiidae) on Machaerium aculeatum (Leguminosae). Revista Brasileira de Botânica, São Paulo, v. 10, p. 117-123, 1987.

FERNANDES, G. W.; TAMEIRÃO NETO, E.; MARTINS, R. P. Ocorrência e caracterização de galhas entomógenas na vegetação do campus pampulha da universidade federal de minas gerais. Revista Brasileira de Zoologia, São Paulo, v. 5, p. 11-29, 1988.

GAGNÉ, R. J. Family Cecidomyiidae. In: PAPAVERO, N. (Org.). A catalogue of the Diptera of the Americas South of the United States. São Paulo: Secretaria da Agricultura. Departamento de Zoologia, 1968. p. 23.1-23.

GAGNÉ, R. J. The plant-feeding gall midges of North America. Ithaca: Cornell University Press, 1989. $356 \mathrm{p}$.

GAGNÉ, R. J. The gall midges of the Neotropical Region. Ithaca: Cornell University Press, 1994. 352 p.

GAGNE, R. J.; SOSA, A.; CORDO, H. A new neotropical species of Clinodiplosis (Diptera: Cecidomyiidae) injurious to alligatorweed, Alternanthera philoxeroides (Amaranthaceae). Proceedings of the Entomological Society of Washington, Washington, DC, v. 106, n. 2, p. 305-311, 2004.

HODGES, C. S. Preliminary exploration for potential biological control agents for Psidium cattleianum. Technical Report, Honolulu, n. 66, p. 32, 1988.

HUENNEKE, L. F.; VITOUSEK, P. M. Seedling and clonal recruitment of the invasive tree Psidium cattleianum: implications for management of native Hawaiian forests. Biological Conservation, Essex, v. 3, n. 3, p. 199-211, 1990.

INSTITUTO BRASILEIRO DE GEOGRAFIA E ESTATÍSTICA (IBGE). Mapa da vegetação do Brasil. Rio de Janeiro, 1988. 1 mapa, 115 x 96 cm. Escala 1:5.000.000.

JULIEN, M. H. Biological control of weeds: a world catalogue of agents and their target weeds. Brisbane: CAB-ACIAR, 1993. 186 p. 
KRAUS, J. E.; SUGIURA, H. C.; CUTRUPI, S. Morfologia e ontogenia em galhas entomógenas de Guarea macrophylla subsp. Tuberculata (Meliaceae). Fitopatologia Brasileira, Brasília, DF, v. 21, n. 3, p. 349-356, 1996.

LARSSON, S.; STRONG, D. R. Ovipositor choice and larval survival of Dasineura marginemtorquens (Diptera, Cecidomyiidae) on resistant and susceptible Salix viminalis. Ecological Entomology, London, v. 17, n. 3, p. 227-232, 1992.

LEGRAND, C. D.; KLEIN, R. M. Mirtáceas: Flora ilustrada catarinense. Itajaí: Herbário Barbosa Rodrigues, 1977. $158 \mathrm{p}$.

LLOYD, C. J.; HUFBAUER, R. A.; JACKSON, A.; NISSEN, S. J.; NORTON, A. P. 2005. Pre and postintroduction patterns in neutral genetic diversity in the leafy spurge gall midge, Spurgia capitigena (Bremi) (Diptera: Cecidomyiidae). Biological Control, Orlando, v. 33, n. 2, p. 153-164, 2005.

MADEIRA, J. A.; MAIA, V. C.; MONTEIRO, R. F. Gall makers (Cecidomyiidae, Diptera) on Calophyllum brasiliense Camb. (Clusiaceae): descriptions and biology. Arquivos-do-Museu-Nacional, Rio de Janeiro, v. 61, n.1, p. 31-48, 2003.

MAIA, V. C. Descrição de duas espécies novas de Cecidomyiidae (Diptera) associadas a Eugenia spp. (Myrtaceae). Revista Brasileira de Entomologia, São Paulo, v. 37, n. 4, p. 717-721, 1993.

MAIA, V. C. The gall midges (Diptera, Cecidomyiidae) from three restingas of Rio de Janeiro State, Brazil. Revista Brasileira de Zoologia, São Paulo, v. 18, n. 2, p. 583-629, 2001.

MAIA, V. C.; MONTEIRO, R. F. Espécies cecidógenas (Diptera, Cecidomyiidae) e parasitóides (Hymenoptera) associadas a Guapira opposita (Vell.) Reitz. (Nyctaginaceae) na restinga da barra de maricá, rio de janeiro. Revista Brasileira de Zoologia, São Paulo, v. 16, n. 2, p. 483-487, 1999.

MAIA, V. C.; AZEVEDO, M. A. P.; COURI, M. New contribution to the knowledge of the gall midges (Diptera, Cecidomyiidae) from the restinga of Barra de Marica (Rio de Janeiro, Brazil). StudiaDipterologica, Germany, v. 9, n. 2, p. 447-452, 2002.

MANI, M. S. Ecology of plant galls. Deen Haag: Dr. W. Junk, 1964. 434 p.

MANI, M. S. Introduction to cecidology. p. 3-7. In: SHORTHOUSE, J. D.; O. ROHFRITSCH. Biology of insect inducing galls. New York: Oxford University Press, 1992. 285 p.

MARKIN, G. P.; NAGAT, R. F.; GARDNER, D. E. Biological control of introduced weeds of native Hawaiian forests. In: CONRAD, C. E.; NEWELL, L. A. Proceedings of the session on tropical forestry for people of the pacific. Honolulu: [s.n.], 1991. XVII Pacific Science Congress, Honolulu, 2728 May.

MEYER, J. Y. Threat of invasive alien plants to native flora and forest vegetation of Eastern Polynesia. Pacific-Science, Honolulu, v. 58, n. 3, p. 357-375, 2004.

NGAKAN, P. O.; YUKAWA, J. Synchronization with host plant phenology and gall site preference of Dinipponaphis autumna (Homoptera, Aphididae). Applied Entomology and Zoology, Tokyo, v. 32, p. 81-90, 1997.

RODERJAN, C. V.; KUNIYOSHI, Y. S.; GALVÃO, F. As regiões fitogeográficas do estado do paraná. Acta forestalia brasiliensis, Brasília, DF, v. , p. 3-7, 1993.

ROHFRITSCH, O. Patterns in gall development. In: SHORTHOUSE, J. D.; O. ROHFRITSCH. Biology of insect inducing galls. New York: Oxford University Press, 1992. p. 60-86.

SKUHRAVÁ, M.; SKUHRAVÝ, V. Biology of gall midges on commom reed in Czechoslovakia. In: SHORTHOUSE, J.; ROHFRITSCH, O. Biology of insect induced galls. New York: Oxford University Press, 1992. p. 196-207. 
SKUHRAVÁ, M.; HINZ, H. L. Rhopalomyia tripleurospermi sp. n. (Diptera: Cecidomyiidae), a new gall midge species on Tripleurospermum perforatum (Asteraceae: Anthemideae) in Europe, and a biological control agent in Canada. Acta-Societatis-Zoologicae-Bohemicae, Czech Republic, v. 64, n. 4, p. 425435,2000

SOBHIAN, R.; LITTLEFIELD, J.; CRISTOFARO, M.; MANN, K. Biology and host specificity of Spurgia capitigena (Bremi) (Dipt., Cecidomyiidae), for the biological control of Euphorbia esula L. in North America. Journal of Applied-Entomology, Berlin, v. 124, n.9/10, p. 333-338, 2000.

STILING, P.; ROSSI, A. M.; STRONG, D. R.; JOHNSON, D. M. Life history and parasites of Asphondylia borrichiae (Diptera: Cecidomyiidae), a gall maker on Borrichia frutescens. Florida Entomologist, Gainesville, v. 75, n. 1, p. 130-137, 1992.

SUÁREZ, E.; CALVO, R. Formacion de agallas en los frutos del guisaro (Psidium guineense SWARTZ) (Myrtaceae) y su relacion con los microhimenopteros Prodecatoma sp. (Eurytomidae) y Torymus $s p$. (Torymidae). Brenesia, San Jose, CR, v. 31, p. 43-52, 1989.

SYRETT, P.; SMITH, L.; GROSSKOPF, G.; MEURK, C. Predicting the likely success of biological control of hawkweeds in New Zealand. Plant Protection Quarterly, Victoria, v. 16, n. 4, p. 172-176, 2001.

TAVARES, J. S. Cecidologia brasileira. As restantes famílias. Braga, Broteria. Série Zoológica, Lisboa, v. 20, p. 5-48, 1922.

VElosO, H. P.; RANGEL-FILHO, A. L.; LIMA, J. C. A. Classificação da vegetação brasileira, adaptada a um sistema universal. Rio de Janeiro: IBGE, 1991. 123 p.

WIRTH, F. F.; DAVIS, K. J.; WILSON, S. B. Florida nursery sales and economic impacts of 14 potentially invasive landscape plant species. Journal of Environmental Horticulture, United States, v. 22 , n. 1, p. 12-16, 2004.

YAHAYA, I. Forest ecosystems of the Comoros: biodiversity, principle threats, prospect for improvement - the case of Kartala forest on Ngazidja island. REGIONAL WORKSHOP ON INVASIVE ALIEN SPECIES AND TERRESTRIAL ECOSYSTEM REHABILITATION IN WESTERN INDIAN OCEAN ISLAND STATES, Seychelles. Proceedings of the... Mauritania: IUCN-The World Conservation Union, 2003. p. 105-118.

YUKAWA, J. Synchronization of galls with host plant phenology. Population Ecology, Tokyo, v. 42, n. 2, p. 105-113, 2000. 